\title{
Study on the Effect of Exogenous Salicylic Acid on Algae Growth in the Environment
}

\author{
Qiuyang $X u^{1}$, M eixue Shi ${ }^{2}$, Shaohua Wang ${ }^{3}$ and $Y$ uchun Qing ${ }^{2 *}$ \\ ${ }^{1}$ Zhoushan giant $Y$ ang) iShu development co Sanya B ranch L td, Sanya, Hainan, 572000, China \\ ${ }^{2}$ Resources and Environment, Hainan Tropical O cean University, Sanya, Hainan, 572000, China \\ ${ }^{3}$ China U rban Construction Research Institute Co Hainan Branch L td, Sanya, Hainan, 572000, China
}

\begin{abstract}
With the development of industry, synthetic drugs and their secondary metabolites bring some hidden dangers to environmental safety. As an excellent drug, salicylic acid is widely used in cardiovascular drugs, industrial dyes, skin care and acne products, etc. Therefore, it is possible to detect salicylic acid molecules, its derivatives or secondary metabolites in the natural environment. It should be noted that salicylic acid, as an endogenous signal molecule, can greatly affect plant growth. In this study, the common algal Spirogyra in fresh water was taken as the research object. The effects of exogenous salicylic acid on the growth of Spirogyra were analyzed through the changes of chlorophyll content and plant cross section during the growth of Spirogyra. It is found that the concentration of salicylic acid in the natural environment generally promotes the development of algae and improves their resistance to environmental stress. Therefore, as a possible correlation index of algal blooms, it deserves our attention.
\end{abstract}

\section{Introduction}

Salicylic acid is widely used as medicine intermediate and cosmetic additive. Therefore, it is easy to find salicylic acid molecules, decomposition products of salicylic acid derivatives and their secondary metabolites in the natural environment. As salicylic acid is a kind of plant endogenous regulator, it can affect plant growth. Charles Claran, an American plant physiologist, has found that salicylic acid has many magical effects on plants. The research has proved that salicylic acid can not only improve the drought tolerance ability of plants, accelerate flowering, but also improve the germination rate of seeds, promote the rooting of cuttings, help plants improve the ability of cold resistance and anti-virus ${ }^{[1-2]}$. The effect of salicylic acid on plants is usually due to the inhibition of glycolate oxidase activity by salicylic acid derivatives decomposition or secondary metabolism, such as acetic acid, which promotes photosynthesis ${ }^{[3-4]}$. In the past, salicylic acid was used to improve the survival rate of plants, promote their robust growth, improve their resistance to adversity, improve the survival rate and prolong their life span ${ }^{[5]}$. And that's one of the reasons.

Unfortunately, we often neglect some important points, such as the bioaccumulation of salicylic acid in the water environment. What is in the soil is often absorbed by plants quickly, unless the amount is too large. Because the water is rich in algae, the intervention of salicylic acid may cause adverse reactions such as algal eruption, which is particularly worthy of our vigilance. The main purpose of this experiment is to study the effect of exogenous salicylic acid input on the growth of algae, and then explore the possible impact on the aquatic environment.

\section{Experimental process and analysis}

In this study, the device used to cultivate the sponge is a cylinder with a diameter of $12 \mathrm{~cm}$ and a height of $20 \mathrm{~cm}$. The reactor device structure is presented in Figure 1. It is equipped with water circulation filtration and aeration device. We use potentiometers to connect pumps of the same kind in series to ensure that they can run synchronously. Of course, we not only use it to cultivate sponge, but also use it as a reactor in the later stage. At the beginning, we used clear water to breed the sponge for several days, and then began to add a proper amount of nutrients every other week to maintain its growth.

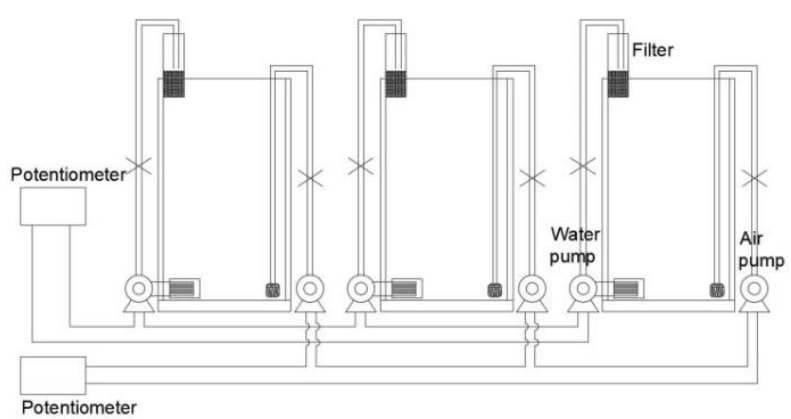

Figure 1. Structure of algal culture plant.

\footnotetext{
*Corresponding author's e-mail: qinyuchun@ 126.com
} 


\section{$2.1 \quad$ Test stage}

Once the trial phase begins, we will not provide any nutrients to the sponge except salicylic acid. We used $0.02 \%$ solution of sodium chloride as the culture medium in the experimental stage to simulate the fresh water environment. Then put $1 \mathrm{~L}$ of culture medium into each device. We take out the water sponge and drain it, then we dry the water with absorbent paper and weigh it and put $50 \mathrm{~g}$ water sponge into each reactor. We prepare $0 \mathrm{mg} / \mathrm{L}-2000 \mathrm{mg} / \mathrm{L}$ salicylic acid solution of different concentrations, and then add it to each reactor from low to high according to the concentration gradient, a total of 9 reactors.

\subsection{Experimental result}

The whole system has been running for 21 days. Every day, we will take out a certain amount of sponge to analysis the change of chlorophyll content and the change of salicylic acid concentration in the culture medium. The amount of salicylic acid consumed by each group of samples over time is shown in Figure 2 below. With the increase of the concentration of salicylic acid in the culture medium, the absorption of a certain amount of sponge tends to a limit value. This value is about $38 \mathrm{mg} / \mathrm{L}$. It shows that the absorption capacity of sponge to salicylic acid is limited in a certain period of time. At this point, it is the same for algae and plants. Under this condition, we can analysis how long for example, plants or algae can eliminate the impact of salicylic acid in the environment, or explore whether they will maintain such absorption activity all the time.

During the cultivation period, we take out a certain amount of water sponge every day for chlorophyll content analysis, the way we use is to use the classical Dual Wavelength Spectrophotometry after ultrasonic extraction ${ }^{[6-7]}$, as is shown in Figure 3 below. When the concentration of salicylic acid is in the range of 50$150 \mathrm{mg} / \mathrm{L}$, the life activity of sponge is effectively maintained. It is shown that salicylic acid can promote the growth of algae in a certain concentration range, which is similar to its effect on plants. When the concentration of salicylic acid is in the range of 50$150 \mathrm{mg} / \mathrm{L}$, compared with the concentration of salicylic acid of $0 \mathrm{mg} / \mathrm{L}$, we found that although at the early stage, the chlorophyll loss rate of the groups with salicylic acid slowed down significantly. In general, the similar effect of salicylic acid on plants is interpreted as that salicylic acid reduces the energy consumption by reducing the sensitivity of plants to the surrounding environment and the life activities of plants. At this point, we think it is also reasonable to put it on algae. When the concentration of salicylic acid was too high, the sponge soon died. The results showed that high concentration of salicylic acid had toxic effect on the growth of sponge cells. From this point of view, the effect of salicylic acid on sponge can be understood as a process similar to stimulation of adaptation. At first, it has a stimulating effect on sponge, just like people eat chili, a little spicy will not make people feel uncomfortable. But the first attempt of a person who has never eaten pepper will definitely make him feel uncomfortable, but he can adapt after a period of time. When salicylic acid continued to stimulate the cell, the resistance of the cell increased. At this time, even though the cells of the cell were damaged due to the toxic effect, the cell was able to survive in a low level of life activity.

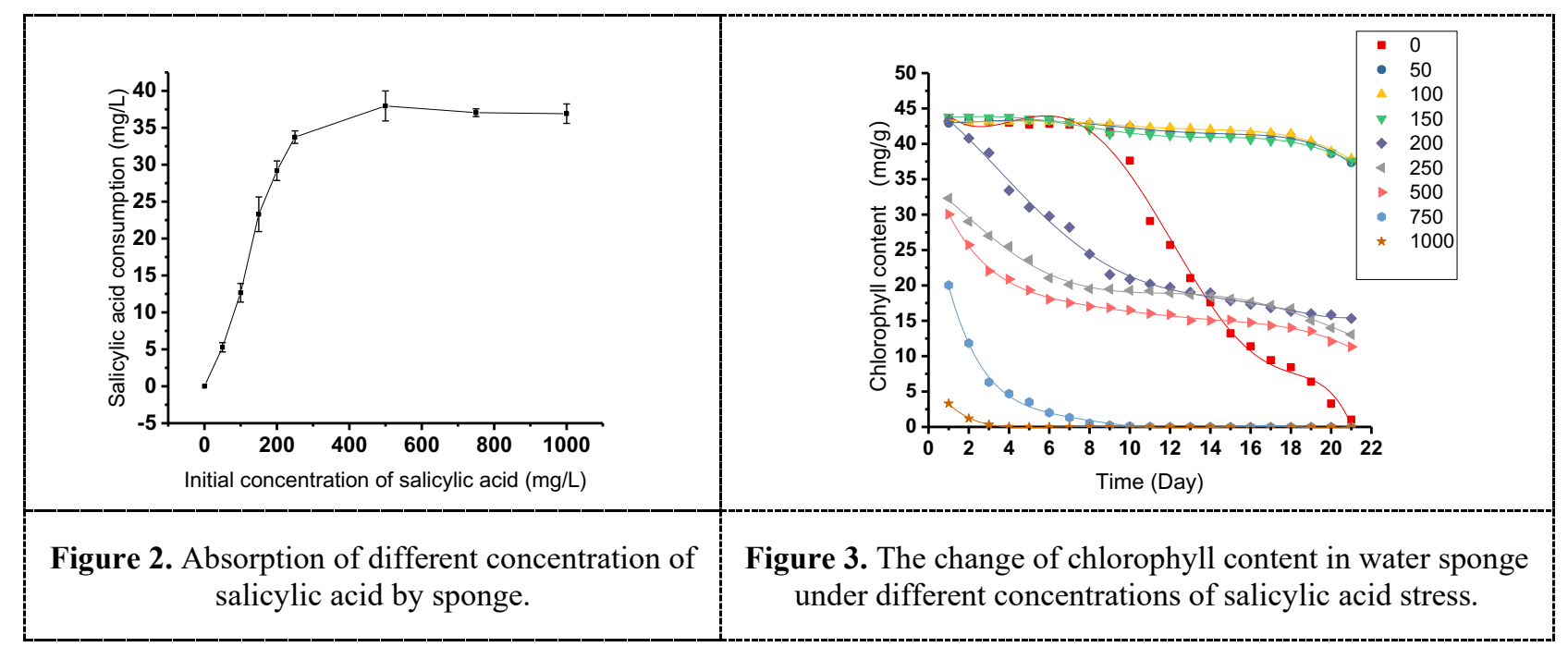

\subsection{Analysis of tissue sections}

When we compare and analyze the sections cultured with high concentration and low concentration of salicylic acid, it is found that salicylic acid crystals coagulate outside the cell wall of high concentration of salicylic acid, so it is speculated that the anoxia caused by crystal covering is also one of the reasons to accelerate the death of sponge. Salicylic acid is one of the carbon sources of Spirogyra, and plays a role in balancing the extracellular pressure of Spirogyra. However, under the stress of salicylic acid, algae can induce the production of active oxygen and lipid peroxidation. When the concentration of aspirin is high, the destruction of algal biofilm will accelerate the internal flow of sodium ions, increase the 
toxicity of ions in algal cells, and accelerate the death of sponge ${ }^{[9]}$. Finally, it was found that the amount of salicylic acid measured after extraction was significantly less than the consumption of salicylic acid in the culture medium. This proves that salicylic acid can also be used as a carbon source by algae, but the consumption is not very large.

\section{Conclusion}

It was found that the main effect of salicylic acid on algae growth was to maintain the growth of Spirogyra and promote the mitosis of algae. In the aspect of promoting algae growth, salicylic acid can improve the adaptability of algae to the surrounding environment by reducing the sensitivity of algae to the surrounding environment through continuous stimulation. According to the analysis of the section of sponge, the micro grains attached to the surface of algal filaments weaken the respiration of algal plants and reduce the nutrient consumption. The low concentration of salicylic acid had little toxic effect on algae, and the growth of sponge was promoted. When the concentration is high but does not reach the short-term level, the loss of chlorophyll will slow down obviously when the sponge adapts to the stimulation of salicylic acid. For one thing, too high concentration of salicylic acid can cause too strong respiratory inhibition, for another, it can also lead to the death of the imbalance of internal and external pressure.

It was found that when the content of salicylic acid in the environment was $50-150 \mathrm{mg} / \mathrm{L}$, it could significantly increase the life of the sponge. When the content of salicylic acid in the environment is higher than $750 \mathrm{mg} /$ $\mathrm{L}$, it will quickly lead to the death of sponge. However, the content of salicylic acid in the normal environment will not reach the level of causing the death of sponge, so most of the salicylic acid in the natural environment will promote the growth and reproduction of algae. Although salicylic acid or its derivatives may not be the reasons which lead to algal blooms, but through experiments, it is not difficult to find that salicylic acid can be used to stimulate and improve the environmental resistance of algae. Therefore, it is possible to use salicylic acid to stimulate freshwater algae or seaweeds, and domesticate varieties that can withstand more severe environment. Or the release of salicylic acid to endangered algae in the environment may also improve their treatment probability. However, if there is a large amount of salicylic acid in the natural environment, it is still worthy of our vigilance. Because they are likely to cause harmful plants to grow wild, after all, they are more competitive than ordinary plants. Therefore, salicylic acid can also be used as one of the reference indicators to indicate algal blooms in water. Of course, more detailed experiments and investigations are needed to further analysis whether salicylic acid will be discharged to cause environmental pollution. It is also necessary to further study the mechanism of the effect of salicylic acid on the growth of algae.

\section{References}

1. Yaqin Long, Wandong Wang, etc. (2009) Salicylic Acid Induced Resistance of Plants against Insects and Diseases and Its Interaction Mechanism. J. Chinese Journal of Tropical Agriculture, 29 (12): 46-50

2. Xuejiao Meng, Kun Di, etc. (2010) Progress of Study on the Physiological Role of Salicylic Acid in Plant. J. Chinese Agricultural Science Bulletin, 26 (15): 207-214

3. Guozhang Kang, Guchou Sun, etc. (2004) Salicylic acid and its environmental stress tolerance in plants. J. Guihaia, 24 (2): 178-183

4. Weifeng Zhao, Yuzhi Yang, etc. (2007) Salicylic acid and the resistance of plant. J. Acta Agriculturae, 23 (2): 108-113

5. Jing Cui. (2007) Effects of Salicylic Acid on Resisting Adversity of Plants. J. Anhui Agri Sci Bull, 13 (9): 35-38

6. Libing Zhang, Qishan Wang, etc. (2008) Discussion on Measurement of Chlorophyll-a in Phytoplank on with Ethanol. J. Environmental Monitoring in China, 24 (6): 9-10

7. Qi Gao, Xuguang Zhao, etc. (2007) Study on the determination of chlorophyll content in Leek by spectrophotometry and ultrasonic extraction. J. Science and Technology of Food Industry, 08 (1): 166-167

8. Dehong Li, Ruizhi Pan, etc. (1995) The role of salicylic acid in plants. J. Plant Physiology Communications, 31 (2): 144-149 